\title{
GESTIÓN DE CALIDAD PARA LA PUESTA EN VALOR DE LOS BAÑOS TERMALES DE CHURÍN EN LA PERSPECTIVA DEL DESARROLLO SOSTENIBLE
}

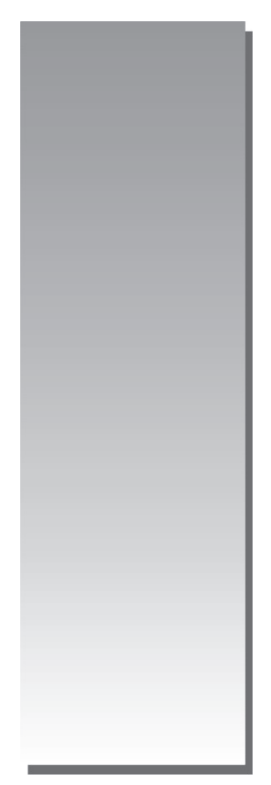

\author{
Niko Cruz Gonzales* \\ nikturismo@hotmail.com
}

\begin{abstract}
RESUMEN
La investigación se titula "Gestión de calidad para la puesta en valor de los baños termales de Churín en la perspectiva del desarrollo sostenible". El objetivo general de la investigación es analizar la gestión de calidad para poner en valor los baños termales de Churín en la perspectiva del desarrollo turístico sostenible. Para cumplir con este objetivo se ha efectuado una revisión bibliográfica sobre los enfoques teóricos de la administración turística y se ha sistematizado la información documental sobre las condiciones naturales, las potencialidades turísticas y la percepción de turistas y pobladores de la localidad sobre la importancia de los mencionados baños termales.

La metodología utilizada ha permitido revisar y evaluar las condiciones y características de los baños termales, así como la valoración de la infraestructura de bienes y servicios prestados a los turistas.

Las conclusiones definen las condiciones teórico-prácticas de una gestión de calidad sobre los diferentes procesos, bienes y servicios, así como la participación de la población como una condición necesaria para hacer sostenible la actividad turística. La percepción de los turistas y de los pobladores expresan las insuficiencias y limitaciones actuales del desarrollo turístico y la necesidad de asumir compromisos políticos para lograr el mejoramiento de la calidad de vida en esta localidad. La puesta en valor de los baños termales y de la construcción de la carretera y otras instalaciones alcanza a un monto de S/. 21'641.457,23.
\end{abstract}

Palabras Claves: Gestión de calidad, administración turística, atractivos naturales, baños termales, puesta en valor, percepción de los turistas y pobladores, desarrollo sostenible.

\section{ABSTRACT}

The investigation is entitled "Management Quality for the value of the bath thermal Churín in the perspective of Sustainable Development". The overall objective of the research is to analyze quality to highlight the bath thermal Churín in a development perspective tourist sustainable. To meet this goal has been made a revision bibliographies approaches you mean Management of the rich tourists and has systematized the information documentary on natural conditions, the potential tourists and perceptions tourists and residents of the town on the importance of the bath springs in this town.

The methodology used made it possible to review and evaluate the conditions and character tics of bath springs, ace as the valuation of its infrastructure in goods and services provided to tourists. The

Doctor en Derecho de la Universidad Nacional Federico Villarreal, Magister de Administración-Universidad Alas Peruanas , Licenciado en Turismo-Universidad San Antonio Abad del Cuzco, Asesor de Proyectos de Inversión Publica y Privada del sector turismo 
findings define the terms of theoretical and practical quality management on the various processes, goods and services as well as people's participation as a necessary condition for sustainable tourism activity. The perception of tourists and residents expressed the shortcomings and limitations of the current tourism development and the need to take on political commitments to achieve improved quality of life in this town. The value of the thermal baths and construction of roads and other facilities to reach a total of S/. 21' 641.457,00.

Keywords: Quality management, tourist administration, natural attractions, thermal baths, putting(put) in value, perception of the tourists and settlers, sustainable development.

\section{INTRODUCCIÓN}

La actividad turística constituye -en la actualidad- el principal renglón económico y social de políticas públicas y privadas en muchos países y regiones. El turismo, según la Organización Mundial del Turismo (OMT), mueve más de 18 millones de turistas que gastan US\$ 110.000 millones anuales, dinamizando el intercambio comercial, la producción y venta de artesanías, desarrollando polos regionales de atracción turística en salud, cultura, religión, ocio, diversión, etc.

En nuestro país, dos millones de turistas dejan US\$ 1.200 como promedio de sus visitas, número que se acrecienta cada día por la cantidad, biodiversidad, multiculturalidad, restos arqueológicos y monumentales y la más extraordinaria variedad de microclimas, paisajes y ecosistemas naturales de nuestro país que causan admiración mundial.

Sin embargo, las condiciones de infraestructura vial, de servicios turísticos, calidad de la atención e intervención de la población en el desarrollo de la actividad turística, dejan mucho que desear. Otros factores como la escasa o deficiente publicidad, insuficiente capacitación de los recursos humanos y la informalidad de muchos servicios turísticos, no estimulan un desarrollo sostenido del turismo.

Por estas consideraciones no se atienden las necesidades de los turistas actuales y de las regiones receptoras con amplia potencialidad turística, ni tampoco se protege y fomenta una gestión de calidad de todos los recursos que puedan satisfacer las necesidades económicas, sociales y estéticas, respetando y preservando simultáneamente la integridad cultural, los procesos ecológicos, la diversidad biológica, los ecosistemas, la atención de salud, recreación y ocio, etc.

Es esta preocupación e interés profesional lo que me ha conducido a desarrollar la presente investigación sobre uno de los atractivos turísticos más importantes para la salud y el bienestar individual que se obtiene como son las visitas a los Baños Termales de Churín, situados a escasa distancia de Lima, sobre los que no existe información ni difusión real sobre sus beneficios y condiciones naturales que promocionen su valor y belleza, dentro de un modelo de desarrollo que mejore permanentemente la calidad de vida de la comunidad y facilite al visitante una experiencia de alto nivel, protegiendo su salud y el medio ambiente.

La presente investigación está dividida en cinco capítulos. En el primer capítulo identificamos el problema objeto de investigación, su importancia y los criterios que justifican su realización, así como los objetivos, hipótesis, variables e indicadores que dan cuenta de la compleja realidad abordada. En el segundo capítulo exponemos el marco teórico, desde el punto de vista de la administración-gestión que da consistencia a los conceptos, enfoques, análisis y comprensión cabal sobre la actividad turística, su tipología, los productos, servicios, empresas y también sobre los límites y críticas reconocidas.

Igualmente exponemos los principios y fundamentos de lo que es una gestión de calidad aplicada al turismo, teniendo en cuenta los modelos de calidad, el diseñó y planificación, así como la reingeniería útil para mejorar una gestión de calidad. Igualmente, se presenta la definición, historia y las estrategias del desarrollo turístico sostenible, remarcando la nueva dimensión de lo que es el desarrollo simultáneo de lo económico, lo social y lo ecológico, que da sostenibilidad a cualquier propuesta de desarrollo, incluyendo a la actividad turística.

En el tercer capítulo se consignan los procedimientos metodológicos que han facilitado la recolección de información empírica, el universo y muestra, las unidades de análisis y los métodos, técnicas e instrumentos utilizados en este proceso de acopio de información.

En el cuarto capítulo y a manera de información diagnóstica, incluimos una descripción de las condiciones generales de Churín, sus principales 
actividades económicas, sus recursos potenciales para la actividad turística como son las fuentes de aguas termales y la percepción que tienen los turistas y los pobladores de esta ciudad sobre el turismo, sus logros, dificultades y opiniones sobre el desarrollo turístico.

Finalmente, en el quinto capítulo expongo la información pertinente a la puesta en valor de los baños termales, así como los alcances de las inversiones a ejecutarse para dar cuenta de una gestión de calidad que contribuya, como insumos, para concretizar una política desarrollo sostenible en esta localidad.

Considero que esta investigación y esta propuesta contribuyen a enriquecer la teoría y práctica de la administración, y de las estrategias necesarias para revalorar, preservar y desarrollar el conjunto de actividades económicas, sociales y culturales de Churín, para el mejoramiento de su calidad de vida y del desarrollo social y cultural, a partir de la explotación racional de sus atractivos turísticos.

\section{MÉTODOS}

\section{Ámbito de investigación}

Churín, balneario serrano de hermosos paisajes, es la capital del distrito de Pachangará, provincia de Oyón del departamento de Lima. Se encuentra ubicado al noreste, a solo $203 \mathrm{~km}$ y a seis horas del centro geodésico del Perú (Plaza de Armas de Lima). A esta ciudad se accede viajando en modernos buses, siguiendo la ruta de la carretera Panamericana Norte, por el desvío del Río Seco, bordeando la parte este de las Lomas de Lachay y continuando por la azucarera de Andahuasi y el histórico pueblo de Sayán de Checta, a $102 \mathrm{~km}$ y a tres horas del pueblo de Huaura, cuna de la independencia del Perú.

El Balneario de Churín se encuentra a una altitud de $2.080 \mathrm{msnm}$, alcanzando su altitud máxima en el nevado de Yarahuayna, a $5.190 \mathrm{msnm}$ y la mínima en el puente Tingo (Tincuy), ubicado a $1.980 \mathrm{msnm}$.

A $210 \mathrm{~km}$ al noreste de Lima, tomando inicialmente la carretera Panamericana Norte y luego un desvío afirmado hacia el este, se encuentra Churín. Desde allí la carretera se interna en los Andes hacia un sinnúmero de poblados tradicionales.

Destacan entre ellos Andajes, famoso por su manjarblanco, y los sitios arqueológicos de
Ninash, Kukun, Antasway y Kuray. Además, en Huacho, frente al imponente nevado Yarahuayna (3.200 msnm), se encuentran los restos preíncas de Antamarca y Chaulín en excelente estado de conservación.

\section{Tipo y nivel de investigación}

El tipo de investigación es aplicativo desde que, a partir de la revisión bibliográfica, documental y de archivos, así como de la propia experiencia directa del autor de la investigación, se ha procedido a presentar el proceso de la oferta y demanda turística de los baños termales de Churín en la perspectiva del desarrollo sostenible.

El nivel de investigación es descriptivo-prepositivo ya que, en primer lugar, se exponen a través de mapas, gráficos y fotografías los atractivos turísticos y, a continuación, se ofrece una propuesta de valoración que debe llevarse a la práctica para poner en valor toda el área espacial de la investigación.

\section{Método y diseño de la investigación}

El método de investigación es múltiple, ya que es cualitativo y cuantitativo porque no solo se utilizará el análisis y la síntesis sino también la inducción-deducción y la concreción abstracción, así como el análisis-síntesis en todo el proceso de elaboración teórica, revisión documental y presentación de los atractivos naturales y culturales de la región.

Partimos de que el pluralismo metodológico es la mejor opción ya que el estudio de una realidad concreta, a manera de un diagnóstico, nos permitió analizar los diversos factores económicos, sociales, culturales y turísticos para formular una propuesta que aborda integralmente todas las condiciones apropiadas según la perspectiva del desarrollo turístico sostenible.

El diseño de investigación es descriptivoprepositivo porque incluye la fase de descripción y también las recomendaciones para superar las condiciones de la relativa oferta-demanda de los servicios turísticos en esta región y así poder sintetizar en una propuesta óptima de desarrollo turístico sostenible.

\section{Universo, población y muestra de la investigación}

El universo de la investigación está determinado por todos los turistas y pobladores del lugar que estuvieron presentes en la semana de 
aplicación de la entrevista (del 15 al 22 de enero del 2008). En este sentido damos por similares al universo y a la población.

No se ha utilizado la técnica del muestreo ya que nuestras unidades de análisis fueron los atractivos turísticos, principalmente los baños termales. Sin embargo, consideramos importante contar con la percepción que tienen los pocos turistas y pobladores que están vinculados al negocio del turismo como transportistas, dueños de restaurantes o de alojamientos, quienes cordialmente respondieron a las preguntas de la entrevista, es por ello que solo se han consignado las respuestas a 15 entrevistas.

\section{RESULTADOS Y DISCUSIÓN}

\section{Técnicas, instrumentos y fuentes de recolección de datos}

Las técnicas de investigación a utilizarse son:

- La revisión documental. Se procedió a revisar toda la información existente en el concejo provincial y distrital, así como la existente en la Región Lima y en el Ministerio de Comercio Exterior y Turismo (MINCETUR), además de revistas especializadas y trabajos monográficos existentes en estas instituciones.

- La entrevista fue aplicada a los turistas y pobladores del lugar que amablemente accedieron a responder.

Los principales instrumentos de investigación son:

- Las Fichas de Estudio, de diverso tipo donde se consignará la información teórica más relevante.

- La Guía de Entrevista (se encuentra como anexo).

Técnicas de procesamiento y análisis de datos recolectados

- Selección y representación informativa. La selección y representación informativa estará referida a la información más actualizada sobre los atractivos turísticos de la región.

- Procesamiento de la información. El diseño del levantamiento de datos en campo y la preparación de la lista de ejecución de actividades consideraron los siguientes aspectos:

- Construcción de los instrumentos de registro de datos.
- Planeamiento de métodos de levantamiento de datos y programación del trabajo en campo en las etapas necesarias.

- Determinación, reclutamiento y preparación del personal en levantamiento de datos en campo y procesamiento de información.

- Aplicación de las entrevistas.

- Tabulación de las entrevistas.

- Recomendaciones generales para el manejo de la información.

- Análisis e interpretación de la información.

Es recomendable establecer un núcleo o comisión de coordinación interinstitucional que lleve a cabo las actividades de negociación, promoción y difusión del programa de de inversiones y de control de calidad de las acciones vinculadas no solo al turismo sino también al desarrollo de las actividades productivas y comerciales. La creación de un Consejo Distrital de Desarrollo podría ser la forma de canalizar estos esfuerzos de coordinación.

Es recomendable capacitar al personal que trabaja en los diversos servicios turísticos, aplicando estrategias de calidad en la gestión, control y mantenimiento de las instalaciones y servicios, como son la gastronomía, las oficinas de transportes, etc.

Se debe buscar la participación multidisciplinaria, que fomente el trabajo en equipo de todos los trabajadores, para analizar y proponer soluciones a los diferentes problemas y dificultades del entorno, creando un clima organizacional propicio para el desarrollo turístico sostenible.

El gobierno local debe promocionar la participación de los pobladores y de sus organizaciones sociales y culturales en la protección y promoción de las actividades turísticas.

Se debe implementar un proceso educativo de concientización turística en toda la población en general, en el personal encargado en turismo y en el turista propiamente dicho. Asimismo, se debe implementar un plan de marketing que contemple objetivos, estrategias, políticas, planes de acción para la difusión, por medios informáticos y audiovisuales de las bondades y beneficios de hacer turismo en Churín. 


\section{CONCLUSIONES}

1. Una gestión de calidad implica valorizar el conjunto de factores, productos y atractivos turísticos en la ciudad de Churín, teniendo como eje a sus baños termales y a los demás atractivos turísticos, y espacios naturales que satisfagan los intereses y motivos de visita de los turistas nacionales y extranjeros.

2. La valorización de las condiciones naturales pasa por identificar, difundir y utilizar las características bio-químicas de los baños termales y de la medicina balneología, enfatizando sus beneficios para la salud y el disfrute de un confort que satisfaga las necesidades y expectativas de los turistas.

3. La percepción de los turistas sobre las calidades de los servicios en su visita a Churín resalta las cualidades beneficiosas de sus aguas termales, principalmente (53\%). Destacan, además, su excelente clima (50\%), el trato de los pobladores (54\%), y su comida (100\%). Pero manifiestan su desagrado por la calidad de los servicios (33\% los considera malos).

4. En la percepción de los pobladores de Churín destacan la necesidad de explotar los centros arqueológicos $(80 \%)$, la creación de un centro de capacitación para los agricultores (73\%), desarrollo de la piscicultura (26\%), la promoción de las fiestas (93\%) y el desarrollo comercial (87\%).

5. La propuesta de gestión de calidad de los baños termales de Churín incluye inversiones en los complejos termales por un valor de S/. 1'641.124,43 y la construcción de la carretera asfaltada a Churín que tiene un costo de S/. 20 millones. Esta inversión se requiere para convertir al balneario y al pueblo en atractivos turísticos de calidad.

\section{LITERATURA CITADA}

Acerenza, Miguel Ángel; (2004) Marketing Hotelero. Editorial Trillas- México.

Ávila Acosta, Roberto. (2001). Metodología de la investigación. Ediciones R.A., Lima - Perú.

Bigne, Enrique. (2000). Marketing de destinos turísticos. Análisis y estrategias de desarrollo. Esic, Madrid.

Bunge, Mario. (1982). La Investigación Científica. Editorial Ariel, Barcelona.

Caballero Romero, Alejandro. (1998). Criterios operativos y prácticos de la investigación científica. Editorial Técnico Científica, Lima.

Caballero Romero, Alejandro. (2000). Metodología de la investigación científica. Editorial Técnico Científica, Lima.

CEPAL. (1999). Indicadores de la actividad turística. Comisión Económica para América Latina y El Caribe, Santiago de Chile.

Fernández Fuster, Lily. (1991). Teoría y técnica del turismo. Editora Nacional, Madrid.

Gironda Guerdes, Gerardo. (2005). La empresa de turismo rural: gestión, administración y calidad. Universidad de Madrid, Madrid.

Gurria Di Bella, Manuel. (1994). Introducción al turismo. Editorial Trillas, México DF.

Koontz, Harold y Weihrich, Heinz. (1997). Administración: una perspectiva global. Décima Edición. Editorial Mc Graw Hill. México DF.

Maraví Lindo Alfonso. (2004). Metodología de la investigación científica. Editorial UIGV, Lima.

PROMUDEH - Opción. (2006). Gestión de programas sociales. OEA, Lima.

Rodríguez de los Ríos. Luis. (2005). Manual de seminario de investigación. Editorial UIGV, Lima. 